\title{
Modeling a Multi-Robot System with Fractional-Order Differential Equations*
}

\author{
Bill Goodwine $^{1}$
}

\begin{abstract}
This paper shows that a fractional-order differential equation may be used to accurately model the dynamic relationship between the first and last generations in a fleet of coordinating robots, even when the individual robots and interconnections have the usual integer-order dynamics. Such a fractional-order model offers the possibility of general applicability, particularly in the case of heterogeneous fleets of robots. Such systems tend to be very high order, and therefore model reduction is useful in modeling, simulation and control. Results are presented for the system considered illustrating that the fractional-order model achieves significant computational savings compared to simulating the full system.
\end{abstract}

\section{INTRODUCTION}

This paper investigates fractional-order modeling for multi-robot coordinated control problems. We study a specific system with a topological structure for the interactions illustrated in Figure 1, which is motivated by a viscoelastic model from the literature [1], [2]. We show that even with linear springs and dampers as interconnection models and robots as dynamic double integrators (all integer-order dynamics), fractional-order effects are present, and hence is a consequence of the interconnection topology. While it is the subject of continued investigation, we believe that fractionalorder dynamics may be very common in formation control of systems of mobile robots and other complex and cyberphysical systems.

Recognizing this fractional-order nature of the dynamics is important for several reasons. First, it leads to a deeper understanding of the system and broadens the "toolbox" of control possibilities for multi-robot systems. Second, it provides for substantial model reduction and computational savings for modeling and controlling the system. Third, when considering loop shaping, large frequency ranges characterized by non-integer order dynamics (non-integer magnitude slopes and non-multiple of $90^{\circ}$ phases) may need to be addressed by fractional-order control methods.

Control of multi-robot systems is a well-studied area in robotics and control with many significant contributions. For example, see [3] (decentralized nearest-neighbor rules), [4] (consensus problems), [5] (graph theory), [6] (potential functions and virtual leaders), [7] (behavior-based), [8] (visionbased formation control) and survey papers [9], [10]. Some of the author's prior work is directed toward exact model reduction for symmetric systems [11]-[13].

*The partial support of the US National Science Foundation under the CPS Large Grant No. CNS-1035655 is gratefully acknowledged.

${ }^{1}$ Bill Goodwine is with Faculty of Aerospace \& Mechanical Engineering, University of Notre Dame, Notre Dame, Indiana 46556 USA billecontrols.ame.nd.edu.

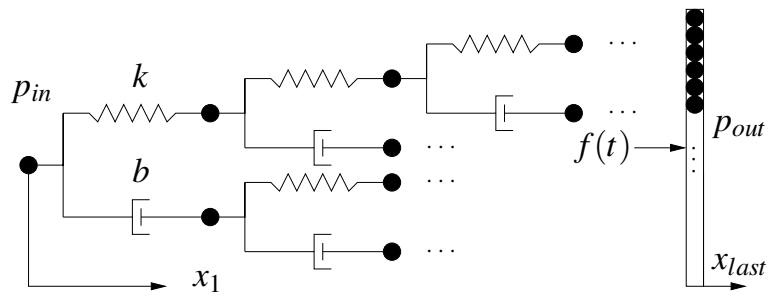

Fig. 1. Structure of robotic formation.

Fractional calculus has a much longer history. As a mathematical subject, it naturally dates back to near the foundations of calculus, and it has been used in engineering and robotic applications for at least several decades. Books on the mathematics and engineering applications include [14], [15] and there are a number of review articles as well [16], [17]. One closely related study is [18], [19] which studied formation control of fractional systems. While involving fractional-order systems and formation control, that paper considered a different problem in that the individual components are fractional in nature; whereas, in this present paper, the fractional dynamics arise from the structure of the interaction among the agents. Other related studies include [20] (walking robots), [21], [22] (flexible manipulators), [23] (time delays) and control using fractional-order PID control [22], [24].

The rest of this paper is organized as follows. Section II introduces a simple multi-robot motivational example with some attributes that suggest fractional-order dynamics. Section III gives an overview of fractional calculus. Section IV verifies that the system from Section II is, indeed, fractionalorder in nature, illustrates the manner in which the dynamics of the system converge to the fractional-order model and presents computational results related to scaling and computational complexity. Finally, Section V presents conclusions and future work.

\section{SYSTEM FORMATION DYNAMICS}

Consider a potential-driven formation control problem, an example appliction of which would be for contamination mapping and control. For example, if toxic waste is spilled at a specific location and has started to diffuse or advect to the surrounding environment, a fleet of robots to map and clean the spill could require that many robots be located where the contamination level is high, and fewer would be required where it is low. When the spread of contamination is very dynamic (such as in a river) or extremely dangerous 


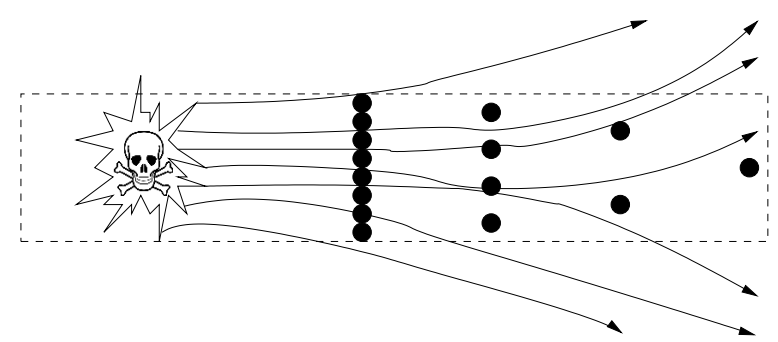

Fig. 2. Schematic of desired robot formation.

(radiation for example), then precise control of the dynamic response of the system is needed.

One way to accomplish such a task could be with a formation with a tree graph structure. In this case, there is one robot at the first generation, two at the second, four at the third, etc., where each successive generation with a greater number of robots is positioned in areas of greater contamination. This scenario is schematically illustrated in Figure 2 where the black dots illustrate the robots and the lines represent the contamination disbursement flow lines. Hence, where the contours are closer together we desire a great number of robots per unit area (to the left) and conversely to the right we need fewer per unit area. Of course in a real-world deployment the robots in each generation would be arranged along the level sets of the contamination concentration, which may have a very complicated shape.

The rest of this paper considers the asymmetric control structure illustrated in Figure 1. We illustrate the interconnections with mechanical components; whereas, obviously for mobile agents these are not mechanical but are implemented by a controller. In each generation the number of robots is doubled. Each robot in the lower generation is related to two in the subsequent generation where the control force between the lower generation robot and one of the next robots is given by a linear spring relationship and the relationship to the other robot is represented by a viscouse damper. We assume all the robots in the final generation are at the same potential and $f(t)$ represents the force on the final generation by all the robots in the penultimate generation.

For clarity of presentation, consider the case where the interior robots have high-gain controllers or small masses and treat their dynamics as algebraic rather than secondorder. In that case [2] shows that the network is self-similar and the transfer function from the difference in force exerted by the first and last generation to how much the network is compressed or extended from equilibrium given by a repeated fraction. Specifically, let

$$
G(s)=\frac{X_{1}(s)-X_{\text {last }}(s)}{F(s)},
$$

where $x_{1}$ denotes the position of the first generation and $x_{\text {last }}$ denotes the position of the last generation. If we let $G_{1}(s)=1 / k$ and $G_{2}(s)=1 /(b s)$, then

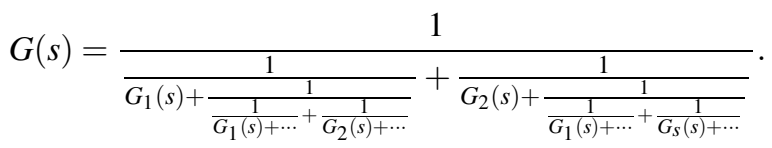

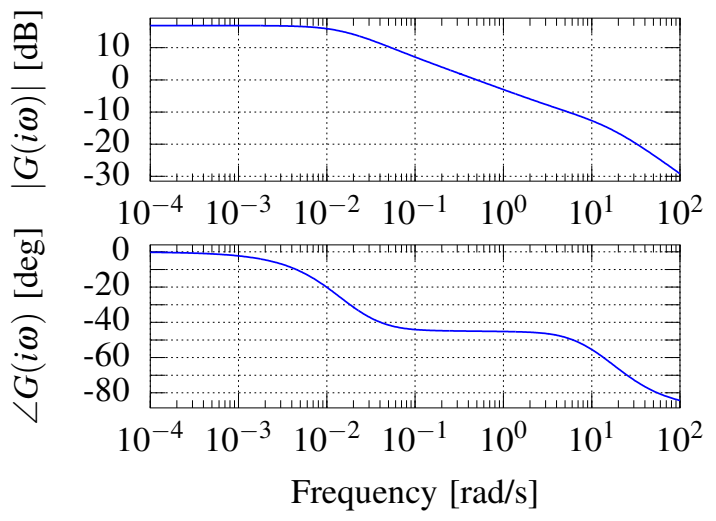

Fig. 3. Network stress-strain frequency response.

This transfer has a denominator with order $2^{N}$ where $N$ is the number of generations in the system. ${ }^{1}$ The frequency response for this transfer function for $k=b=1$ and for 7 generations is illustrated in Figure 3.

The important feature of Figure 3 is that there is a large frequency band over which the slope of the magnitude plot is $-10 \mathrm{db} /$ decade and the phase is $-45^{\circ}$. Naturally, this leads us to consider to what extent this system can be approximated by a transfer function containing a term of the form $\sqrt{s}$, or equivalently, in the time domain, a derivative of order $1 / 2$, which is essentially the focus of the rest of this paper.

To understand the fractional-order nature of the Bode plot, Figure 4 illustrates the pole-zero map for this system with 5 generations. The poles and zeros are evenly spaced (logarithmically), so that half way between any two poles is a zero. Hence as frequency is increased, any pole that adds $-20 \mathrm{db} /$ decade to the slope is offset by a zero half way to the next pole, so if they are closely spaced over a broad frequency range, a persistent range with a slope of $-10 \mathrm{db} /$ decade results. ${ }^{2}$ Clearly, this mechanism can to lead to just about any slope and could be constructed by varying the spacing between the poles and zeros.

\section{FRACTIONAL-ORDER DIFFERENTIAL EQUATIONS}

It is, of course, natural to ask, given a function, $f(t)$ with a first derivative, $f^{(1)}(t)$ and second derivative, $f^{(2)}(t)$, whether there are operators "in between" the integer order derivatives such as

$$
\frac{d^{\frac{1}{2}}}{d t^{\frac{1}{2}}} f=f^{\left(\frac{1}{2}\right)}
$$

which generalizes the notion of an integer-order derivative. We start with $f(t)=t^{k}$, and observe that

$$
\frac{d^{n}}{d t^{n}} t^{k}=\frac{k !}{(k-n) !} t^{k-n}
$$

\footnotetext{
${ }^{1}$ This can be considered a stress-strain relationship because it relates how much the network is compressed or extended to the difference in force exerted on the first and last elements.

${ }^{2}$ The pole-zero map for the 7-generation network is similar, but has many complex pole-zero pairs, which makes the interpretation less transparent, but it is essentially the same.
} 


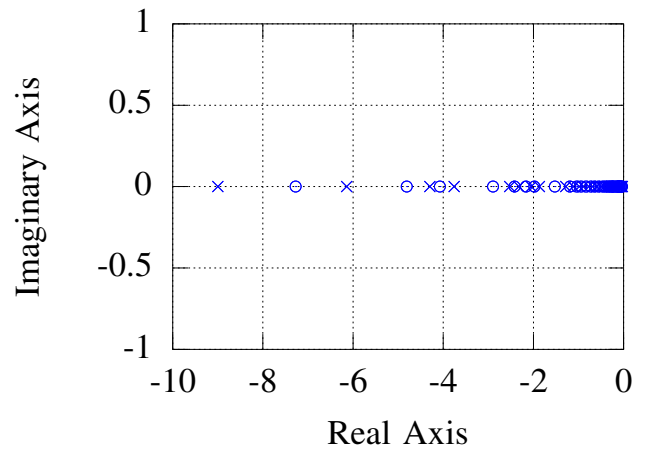

Fig. 4. Pole-zero map for 5 generation network.

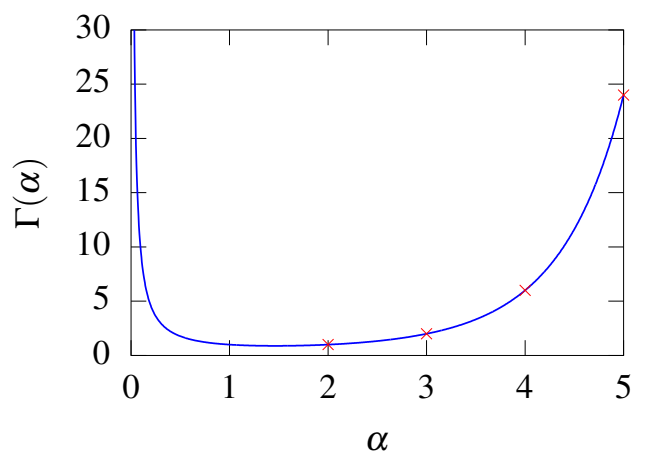

Fig. 5. Gamma function (line) and the first several values of $(n-1) !(\times)$.

for the usual case where $n$ is an integer. Recall that a generalization of the factorial function is the gamma function defined by by

$$
\Gamma(\alpha)=\int_{0}^{\infty} e^{-t} t^{\alpha-1} d t
$$

and illustrated in Figure 5. In the case where $\alpha$ is an integer, this can be integrated by parts multiple times to eliminate the $t$-term in the integrand and it is clear that $\Gamma(n)=(n-1)$ ! which are indicated by the $\times$ marks in Figure 5 .

Replacing the factorials in Equation 2 with gamma functions gives

$$
\frac{d^{\alpha}}{d t^{\alpha}} t^{k}=\frac{\Gamma(k+1)}{\Gamma(k+1-\alpha)} t^{k-\alpha},
$$

which provides a nice generalization of the derivative, as is illustrated in Figure 6 for several $\alpha \in[0,1]$ for $f(t)=t^{2}$.

To extend this notion beyond simple polynomials, consider Cauchy's formula for repeated integration, which is given by

$$
\begin{aligned}
& \int_{0}^{t} \int_{0}^{\tau_{1}} \cdots \int_{0}^{\tau_{n-1}} f\left(\tau_{n}\right) d \tau_{n} d \tau_{n-1} \cdots d \tau_{1}= \\
& \frac{1}{(n-1) !} \int_{0}^{t}(t-\tau)^{n-1} f(\tau) d \tau
\end{aligned}
$$

and is easily proven by induction.

One interpretation of this formula is that "integrating the function, $f, n$ times" is given by the single integral on the right-hand side of Equation 3. In that expression, the number

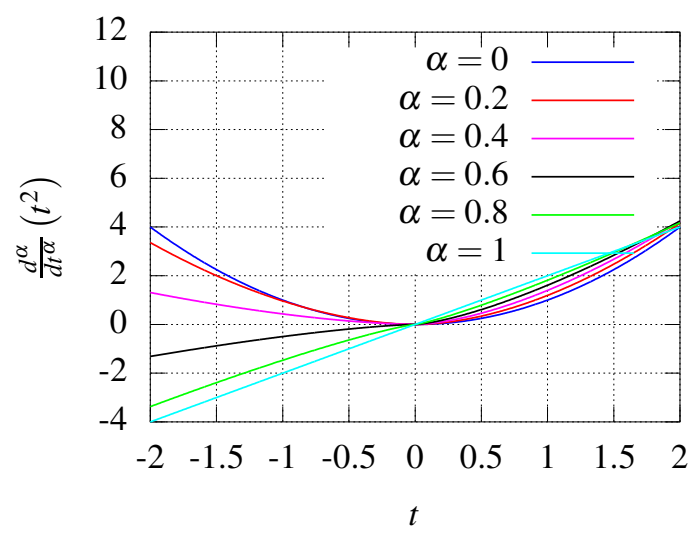

Fig. 6. Fractional-order derivatives for $f(t)=t^{2}$ for various orders between 0 and 1. Note that the zeroth derivative is the parabola, the first derivative the expected straight line and the fractional derivatives between these two vary in a reasonably expected manner.

of integrations, $n$, only appears in the factorial function and in the exponent in the integrand. Of these two, only the factorial function requires $n$ to be an integer. Hence, if we denote $n$ such integrations by $f^{-n}(t)$, we can write

$$
f^{-\alpha}(t)=\frac{1}{\Gamma(\alpha)} \int_{0}^{t}(t-\tau)^{\alpha} f(\tau) d \tau,
$$

which provides a means for fractional-order integration, from which fractional-order derivatives immediately follow. Important complications arise, but the easiest way to see this if, for example, if we want the 2.3 derivative, we integrate 0.7 times and then differentiate (normally) 3 times.

It is worth emphasizing that, unlike integer-order derivatives, fractional-order derivatives require more than local information. In fact, it is apparent from the integral in the definition in Equation 4, that all past values of a function enter into the computation for the fractional derivative. Here we will take a standard linear control-theoretic approach and assume that all initial conditions are zero and also that all the history for all signals for negative times are zero as well.

While closed-form solutions for fractional-order differential equations do exist, we also must resort to numerical approximations. To that end, if we consider the first and second derivatives of a function to be defined as

$$
\begin{aligned}
\frac{d f}{d t}(t) & =\lim _{\Delta t \rightarrow 0} \frac{f(t)-f(t-\Delta t)}{\Delta t} \\
\frac{d^{2} f}{d t^{2}}(t) & =\lim _{\Delta t \rightarrow 0} \frac{f(t)-2 f(t-\Delta t)+f(t-2 \Delta t)}{(\Delta t)^{2}}
\end{aligned}
$$

or in general for an integer $n$

$$
\frac{d^{n} f}{d t^{n}}(t)=\lim _{\Delta t \rightarrow 0} \frac{\sum_{0 \leq m \leq n}(-1)^{m}\left(\begin{array}{c}
n \\
m
\end{array}\right) f(t+(n-m) \Delta t)}{(\Delta t)^{n}}
$$

where the usual binomial coefficient is given by

$$
\left(\begin{array}{c}
n \\
m
\end{array}\right)=\frac{n !}{m !(n-m) !}
$$




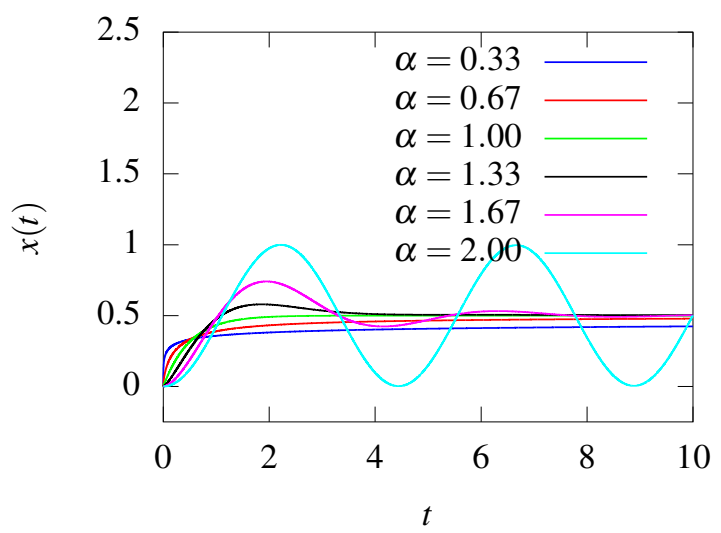

Fig. 7. Solution to Equation 5 using Equation 6.

which, consistent with what we have done so far is easily generalized to non-integers by gamma functions

$$
\left(\begin{array}{c}
\alpha \\
m
\end{array}\right)=\frac{\Gamma(\alpha+1)}{\Gamma(m+1) \Gamma(\alpha-m+1)} .
$$

Using this we arrive at the Grünwald - Letnikov derivative:

$$
\frac{d^{\alpha} f}{d t^{\alpha}}(t)=\lim _{\Delta t \rightarrow 0} \frac{1}{(\Delta t)^{\alpha}} \sum_{j=0}^{\infty}(-1)^{j}\left(\begin{array}{c}
\alpha \\
j
\end{array}\right) f(t+(\alpha-j) \Delta t),
$$

which, similar to Equation 4 includes all past values of $f(t)$. If $\Delta t \ll 1$ and $t=m \Delta t$, then the time shift by $\alpha$ is small and if all the initial conditions are zero, then we have

$$
\frac{d^{\alpha} f}{d t^{\alpha}}(t) \approx \frac{1}{(\Delta t)^{\alpha}} \sum_{j=0}^{m}(-1)^{j}\left(\begin{array}{c}
\alpha \\
j
\end{array}\right) f(t-j \Delta t)
$$

which is a useful approximation to solve fractional-order differential equations.

For example, for

$$
\frac{d^{\alpha} x}{d t^{\alpha}}(t)+2 x(t)=1
$$

substituting the finite-difference approximation from the Grünwald - Letnikov definition and letting $t=m \Delta t$, then

$$
\frac{d^{\alpha} x}{d t^{\alpha}}(m \Delta t)+2 x(m \Delta t)=1
$$

is approximated by

$$
\frac{1}{(\Delta t)^{\alpha}} \sum_{j=0}^{m}(-1)^{j}\left(\begin{array}{c}
\alpha \\
j
\end{array}\right) x((m-j) \Delta t)+2 x(m \Delta t)=1 .
$$

Solving for $x(m \Delta t)$ gives

$$
x(m \Delta t) \approx \frac{1-\frac{1}{(\Delta t)^{\alpha}} \sum_{j=1}^{m}(-1)^{j}\left(\begin{array}{c}
\alpha \\
j
\end{array}\right) x((m-j) \Delta t)}{2+\frac{1}{(\Delta t)^{\alpha}}} .
$$

Solutions for various $\alpha \in[0.25,2.0]$ are illustrated in Figure 7. When $\alpha=1$ and 2 we observe the expected exponential and harmonic solutions, respectively. Intermediate values for the order of the derivative produce reasonably intuitive intermediate responses.

Octave code computing these solutions is:

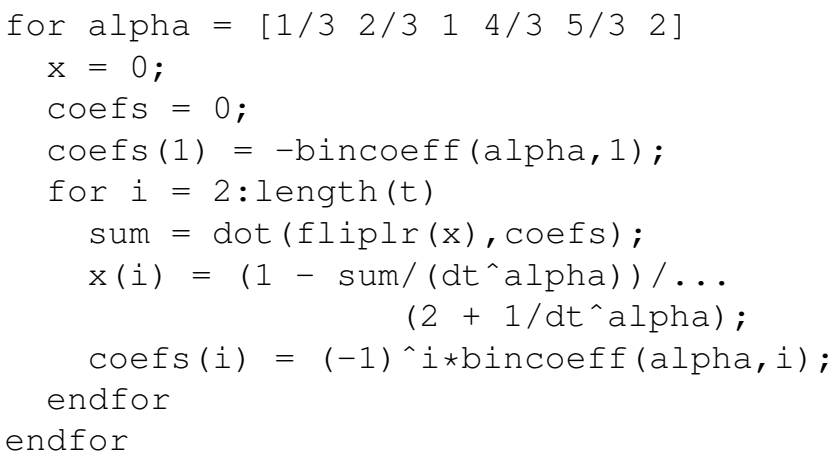

\section{RESULTS}

Returning to the robot formation control problem, note that in the limit of an infinite number of generations, the transfer function in Equation 1 may be written as [2]

$G_{\infty}(s)=\frac{1}{\frac{1}{G_{1}(s)+G_{\infty}(s)}+\frac{1}{G_{2}(s)+G_{\infty}(s)}}=\sqrt{G_{1}(s) G_{2}(s)}=\sqrt{\frac{1}{k b s}}$.

This transfer function clearly will have a Bode plot comprised of a magnitude plot that is a straight line with slope of $-10 \mathrm{db} /$ decade and a constant phase of $-45^{\circ}$, i.e., the entire Bode plot will look like the interesting parts of Figure 3.

Hence, in the limit, the network produces a fractionalorder relationship between the positions of the first and last robots given by

$$
\frac{X_{1}(s)-X_{\text {last }}(s)}{F(s)}=\left(\frac{1}{\sqrt{k b}}\right) \frac{1}{\sqrt{s}} .
$$

If we take as the input to the network the position of the first robot, then we have

$$
m_{\text {last }} s^{2} X_{\text {last }}(s)=\left(X_{1}(s)-X_{\text {last }}(s)\right) \sqrt{k b s}
$$

or the fractional-order transfer function

$$
\frac{X_{\text {last }}(s)}{X_{1}(s)}=\frac{\sqrt{k b s}}{m_{\text {last }} s^{2}+\sqrt{k b s}},
$$

which can be represented by the reduced system illustrated in Figure 8 . This can be represented in the time domain by

$$
m \frac{d^{2} x_{\text {last }}}{d t^{2}}(t)+\sqrt{k b} \frac{d^{\frac{1}{2}} x_{\text {last }}}{d t^{\frac{1}{2}}}(t)=\sqrt{k b} \frac{d^{\frac{1}{2}} x_{1}}{d t^{\frac{1}{2}}}(t) .
$$

Using the Grünwald - Letnikov definition for the fractional derivatives and solving for $x_{\text {last }}(t)$ gives the following numerical approximation at $t=n \Delta t$ :

$$
\begin{aligned}
x_{\text {last }}(n \Delta t) & \approx\left(\frac{1}{\frac{m}{(\Delta t)^{2}}+\frac{\sqrt{k b}}{\sqrt{\Delta t}}}\right) \\
& \times\left[\frac{m}{(\Delta t)^{2}}(2 x((n-1) \Delta t)-x((n-2) \Delta t))\right. \\
& -\sum_{j=1}^{n}(-1)^{j}\left(\begin{array}{c}
\frac{1}{2} \\
j
\end{array}\right) x_{\text {last }}((n-j) \Delta t) \\
& \left.+\frac{\sqrt{k b}}{\sqrt{\Delta t}} \sum_{j=0}^{n}(-1)^{j}\left(\begin{array}{c}
\frac{1}{2} \\
j
\end{array}\right) x_{1}((n-j) \Delta t)\right] .
\end{aligned}
$$




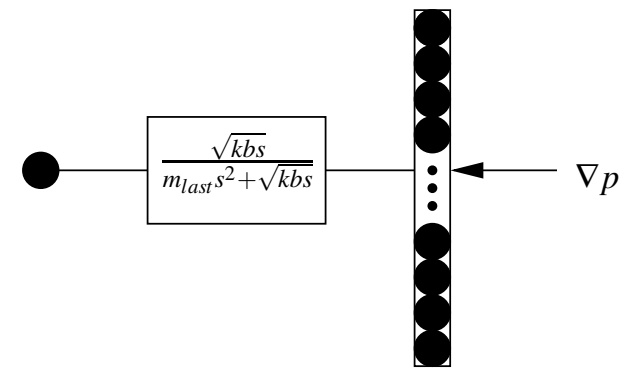

Fig. 8. Fractional order transfer function between first and last generations in the robotic formation.

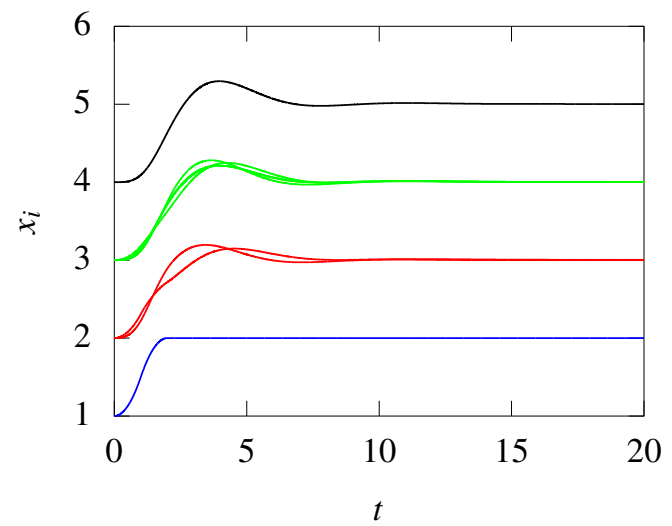

Fig. 9. Comparison of fractional-order solution with full solution for $k=$ $b=1$ and four generations.

Now we consider to what extent a finite network is characterized by such fractional-order dynamics. If the fractionalorder representation is only true in the limit, then this paper is only of academic interest. If it is accurate for a relatively small number of generations, then it is of practical control and robot fleet design importance.

We specify the acceleration profile of the first robot as

$$
\frac{d^{2} x_{1}}{d t^{2}}(t)= \begin{cases}1, & 0<t \leq 1 \\ -1, & 1<t \leq 2 \\ 0, & t>2\end{cases}
$$

and numerically solve for the solution of $x_{\text {last }}$. In the case where $k=b=1, \Delta t=0.005$ and there are only four generations, the solution for all 8 robots is illustrated in Figure 9. In the figure, it is assumed that the uncompressed length of the springs is one and each generation starts from rest at the equilibrium configuration. A comparison of the fractionalorder solution and the full solution for the last robot is illustrated in Figure 10. Clearly the match is excellent.

The accuracy of the fractional-order approximation is dependent on the numerical values chosen for the simulation. Taking $k=1, b=1 / 4, \Delta t=0.005$ and varying the number of generations, a comparison of the full solution and the fractional-order solution is illustrated in Figure 11, where the convergence of the fractional-order solution to the full solution as the number of generations increases is apparent.

To study the computational aspects of the problem, the simulations were performed on a HP DL165 G6 server with

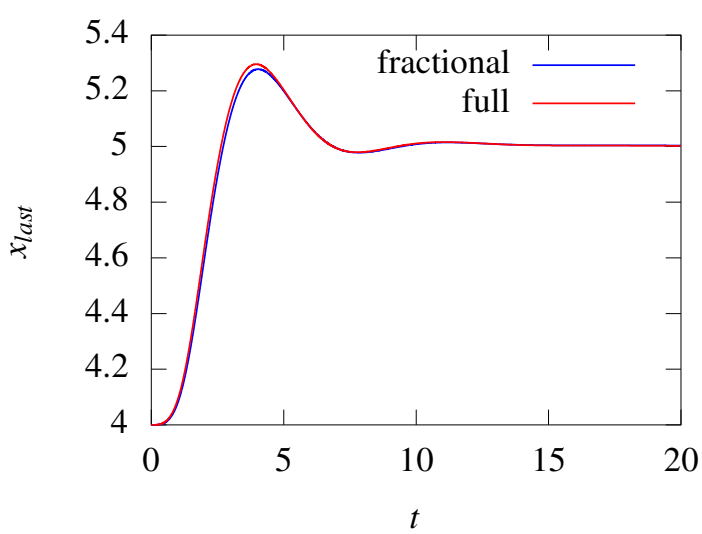

Fig. 10. Comparison of fractional-order solution with full solution for $k=b=1$ and four generations.

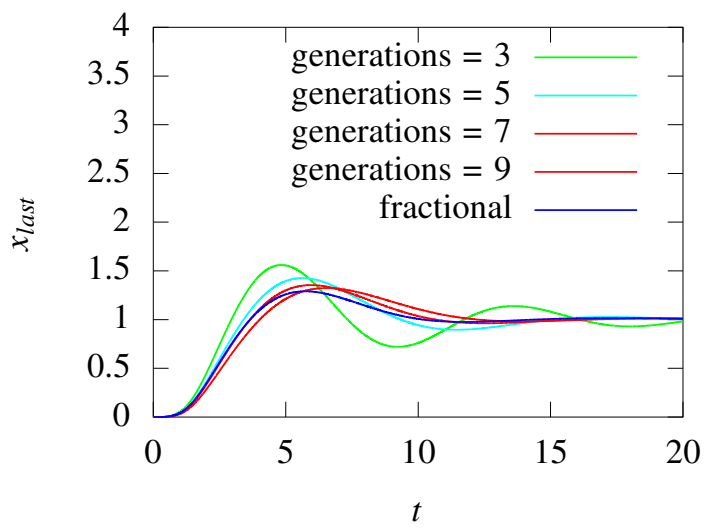

Fig. 11. Convergence to fractional-order solution for $k-1, b=1 / 4, \Delta t=$ 0.005 and various numbers of generations.

dual six-core 2.4 GHz AMD Opteron processors (Model 2431), 12 cores per node and 12 GB RAM. The software used was the Isode () function in octave for the full solutions and an octave script similar to that illustrated previously for the fractional-order solution. The computation times are in seconds and were reported by the octave cputime() function and are illustrated in Figure 12 and summarized in Table I. As is apparent from the table, adding one generation, which doubles the number of robots and interconnections, roughly increases the computation time by a factor of four. For the problem at hand, the fractional-order computation becomes more efficient for the system with more than four generations.

\section{CONCLUSIONS AND FUTURE WORK}

This paper studied the accuracy and computational aspects of modeling a multi-robot system using fractional-order differential equations. We showed that even for a relatively small system comprised of simple elements with integerorder dynamics, the resulting relationship between the first and last generation of robots exhibited significant fractionalorder effects. This was demonstrated both in a frequencyresponse analysis as well as simulations comparing the full integer-order solutions and a fractional-order approximation. 


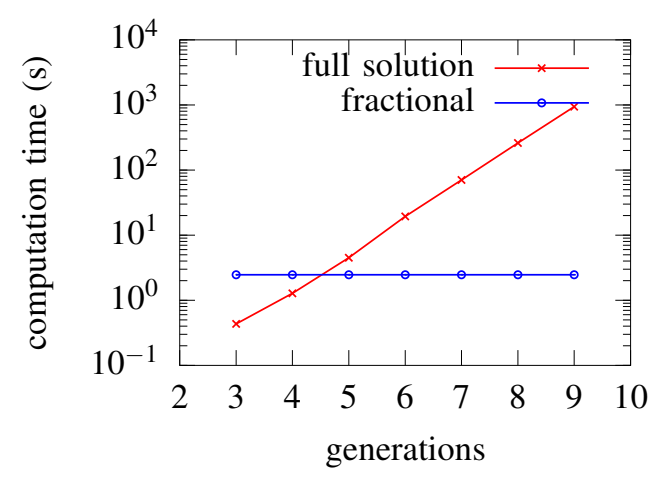

Fig. 12. Computation time for full solution and fractional-order solutions for various numbers of generations.

\begin{tabular}{|c|c|c|}
\hline Generations & Full Simulation (s) & Fractional Simulation (s) \\
\hline 3 & .43393 & 2.4596 \\
4 & 1.2768 & 2.4596 \\
5 & 4.4983 & 2.4596 \\
6 & 19.398 & 2.4596 \\
7 & 70.408 & 2.4596 \\
8 & 261.22 & 2.4596 \\
9 & 938.74 & 2.4596 \\
10 & 3769.9 & 2.4596 \\
11 & 14822 & 2.4596 \\
\hline
\end{tabular}

TABLE I

COMPUTATION TIMES FOR DIFFERENT NETWORK SIZES.

The contributions of this work is putting forth the fractionalorder model as a useful tool in the multi-robot control area, which has been relatively unstudied.

Future work left to do is substantial. While the mere presence of fractional-order dynamics is important for full understanding of the problem, if we are to effectively control such systems, incorporating the fractional-order effects into control design is necessary. Fractional-order control is not new (fractional PID, for example), but it is new in the present context and the nature of the problem naturally leads to the question of fractional-order loopshaping. Another important area is related to fractional-order system identification for complex multi-robot and cyber-physical systems. In our view, the largest open question is to what extent fractional-order dynamics are present in general for multiagent systems? This paper illustrated the fact that fractionalorder effects can arise simply from the interconnections, and complex and cyber-physical systems are characterized, in general, with such structure. If such effects are relatively commonplace, then fractional-order modeling may have a huge impact and be recognized more significantly by the mainstream engineering communities.

\section{REFERENCES}

[1] N. Heymans and J.C. Bauwens. Fractal rheological models and fractional differential equations for viscoelastic behavior. Rheologica Acta, 33:210219, 1994.

[2] Jayson Mayes. Reduction and Approximation in Large and Infinite Potential-Driven Flow Networks. $\mathrm{PhD}$ thesis, University of Notre Dame, 2012.
[3] Ali Jadbabaie, Jie Lin, and A. Stephen Morse. Coordination of groups of mobile autonomous agents using nearest neighbor rules. IEEE Transactions on Automatic Control, 48(6):988-1001, 2003.

[4] Wei Ren, Randal W. Beard, and Ella M. Atkins. Information consensus in multivehicle cooperative control. IEEE Control Systems Magazine, pages 71-82, April 2007.

[5] J. Alexander Fax and Richard M. Murray. Information flow and cooperative control of vehicle formations. IEEE Transactions on Automatic Control, 49(9):1465- 1476, 2004.

[6] N.E. Leonard and E. Fiorelli. Virtual leaders, artificial potentials, and coordinated control of groups. In Proceedings of the 40th IEEE Conference on Decision and Control, pages 2968-2973, December 2001.

[7] Tucker Balch and Ronald C. Arkin. Behavior-based formation control for multirobot teams. IEEE Transactions on Robotics and Automation, 14(6):926-939, 1998.

[8] Aveek K. Das, Rafael Fierro, Vijay Kumar, James P. Ostrowski, John Spletzer, and Camillo J. Taylor. A vision-based formation control framework. IEEE Transactions on Robotics and Automation, 18(5):813-825, 2002.

[9] Richard M Murray. Recent research in cooperative control of multivehicle systems. Journal of Dynamic Systems Measurement and Control, 129(5):571, 2007.

[10] Yongcan Cao, Wenwu Yu, Wei Ren, and Guanrong Chen. An overview of recent progress in the study of distributed multi-agent coordination. Industrial Informatics, IEEE Transactions on, 9(1):427-438, 2013.

[11] M. Brett McMickell and Bill Goodwine. Reduction and non-linear controllability of symmetric distributed systems. International Journal of Control, 76(18):1809-1822, 2003.

[12] M. Brett McMickell and Bill Goodwine. Motion planning for nonlinear symmetric distributed robotic formations. The International journal of robotics research, 26(10):1025-1041, 2007.

[13] Bill Goodwine and Panos J. Antsaklis. Multi-agent compositional stability exploiting system symmetries. Automatica, pages 3158-3166, 2013.

[14] Dumitru Baleanu, Jos Antnio Tenreiro Machado, and Albert C. J. Luo. Fractional Dynamics and Control. Springer Publishing Company, Incorporated, 2011.

[15] Manuel Duarte Ortigueira. Fractional Calculus for Scientists and Engineers, volume 84 of Lecture Notes in Electrical Engineering. Springer, 2011.

[16] M.D. Ortigueira. An introduction to the fractional continuous-time linear systems: the 21st century systems. Circuits and Systems Magazine, IEEE, 8(3):19-26, 2008.

[17] J. Tenreiro Machado, Virginia Kiryakova, and Francesco Mainardi. Recent history of fractional calculus. Communications in Nonlinear Science and Numerical Simulation, 16(3):1140 - 1153, 2011.

[18] Yongcan Cao and Wei Ren. Distributed formation control for fractional-order systems: Dynamic interaction and absolute/relative damping. Systems \& Control Letters, 59(34):233 - 240, 2010.

[19] Yongcan Cao, Yan Li, Wei Ren, and Yang Quan Chen. Distributed coordination of networked fractional-order systems. Systems, Man, and Cybernetics, Part B: Cybernetics, IEEE Transactions on, 40(2):362370, 2010.

[20] Manuel F Silva, JA Tenreiro Machado, and AM Lopes. Fractional order control of a hexapod robot. Nonlinear Dynamics, 38(1-4):417433, 2004.

[21] H. Delavari, P. Lanusse, and J. Sabatier. Fractional order controller design for a flexible link manipulator robot. Asian Journal of Control, 15:783795, 2013.

[22] Chunna Zhao, Dingyu Xue, and YangQuan Chen. A fractional order pid tuning algorithm for a class of fractional order plants. In Proceedings of the IEEE International Conference on Mechatronics \& Automation, 2005.

[23] YangQuan Chen and Kevin L. Moore. Analytical stability bound for a class of delayed fractional-order dynamic systems. Nonlinear Dynamics, 29(1-4):191-200, 2002.

[24] Concepcin A. Monje, Blas M. Vinagre, Vicente Feliu, and YangQuan Chen. Tuning and auto-tuning of fractional order controllers for industry applications. Control Engineering Practice, 16(7):798 - 812, 2008. 